\title{
GEOMETRIC FINITENESS OF CERTAIN KLEINIAN GROUPS
}

\author{
G. P. SCOTT AND G. A. SWARUP \\ (Communicated by Frederick R. Cohen)
}

\begin{abstract}
If $G$ is a discrete subgroup of PSL $(2 ; \mathbf{C})$ representing a fibred 3manifold and $H$ the subgroup of $G$ corresponding to the fibre, we show that any finitely generated subgroup of infinite index in $H$ is geometrically finite.
\end{abstract}

We will prove that if $K$ is a finitely generated subgroup of infinite index in the fundamental group $H$ of the fibre of a hyperbolic 3-manifold $M$ of finite volume, then $K$ is geometrically finite. Work of Bonahon [Bo] and Thurston $\left[\mathrm{Th}_{2}\right]$ implies that the hypothesis that $M$ be a bundle can be weakened. The result one obtains is that if $K$ is a finitely generated subgroup of infinite index in $H$, and if $H$ is a geometrically infinite surface group contained in the fundamental group of a geometrically finite hyperbolic 3-manifold $M$, then $K$ is geometrically finite. For it follows from [Bo] and $\left[\mathrm{Th}_{2}\right]$ that $M$ is finitely covered by $M^{\prime}$ which is a bundle with fibre whose fundamental group is commensurable with $H$. The proof of our result depends on a theorem of Cannon and Thurston [C-T], which we will now describe. Let $G$ be a discrete subgroup of $\operatorname{ISO}\left(H^{3}\right)$ so that $M=H^{3} / G$ is a fibre bundle over the circle and let $H$ be the subgroup of $G$ corresponding to the fibre $F$. Let $S$ be a hyperbolic 2-manifold homeomorphic to the fibre $F$ and let $h: S \rightarrow M$ denote the homeomorphism into $M$. We will identify $\pi_{1}(S)$ with a subgroup $H^{\prime}$ of ISO $\left(H^{2}\right)$ and thus $h_{*}$ is an isomorphism of $H^{\prime}$ onto $H$. The map $h: S \rightarrow M$ lifts to a map $\tilde{h}: H^{2} \rightarrow H^{3}$ and we consider the compactifications $B^{2}=H^{2} \cup S_{\infty}^{1}$ and $B^{3}=H^{3} \cup S_{\infty}^{2}$. The main theorem of [C-T] asserts that when $S$ is closed there is an equivariant extension $j: B^{2} \rightarrow B^{3}$ of $\tilde{h}$. The case when $S$ is not closed but has finite area has been considered by Fenley [F]. We will denote by $j_{\infty}$ the restriction of $j$ to $S_{\infty}^{1}$. Then $j_{\infty}$ is a space-filling curve in the 2-sphere $S_{\infty}^{2}$. With the above notations, our result follows.

Received by the editors August 4, 1989 .

1980 Mathematics Subject Classification (1985 Revision). Primary 30F40, 55A05.

Key words and phrases. Kleinian group, geometric finiteness fibre bundle over the circle, stable and unstable laminations.

The first author's research was partially supported by NSF grant DMS 8702519 . 
1. Theorem. Let $K$ be a finitely generated subgroup of infinite index in $H$, the fundamental group of the fibre of $M$. Then $K$ is geometrically finite.

We will denote by $K^{\prime}$ the subgroup $h_{*}^{-1}(K)$ of $H^{\prime}$. If $\Lambda\left(K^{\prime}\right)$ and $\Lambda(K)$ denote the limit sets of $K^{\prime}$ and $K$ in $S_{\infty}^{1}$ and $S_{\infty}^{2}$ respectively, we have $j_{\infty}\left(\Lambda\left(K^{\prime}\right)\right)=\Lambda(K)$ since the map $j$ is equivariant. The proof will be by examining the map $j_{\infty}$ to show that $\Lambda(K)$ does not fill up $S_{\infty}^{2}$. If there are no parabolics in $K$, this is enough by $[M]$. In the general case one has to verify the evenly cusped condition of $[M]$ to assert geometric finiteness. However, there is additional topological information in our case that makes this verification unnecessary. For, let $M_{H}$ and $M_{K}$ be the covers of $M$ corresponding to $H$ and $K$ respectively. The main result of [Sc1], see also [Sc2], shows that $K^{\prime}$ is a geometric subgroup of some subgroup $H^{\prime \prime}$ of finite index in $H^{\prime}$, i.e., $K^{\prime}$ is the fundamental group of a subsurface of the finite cover of $F$ whose fundamental group is $H^{\prime \prime}$. Thus we can assume, without loss of generality, that $K^{\prime}$ is a geometric subgroup of $H^{\prime}$. It is immediate that $M_{K}$ has only one end modulo the parabolics. That is, by removing open neighborhoods of cusps in $M_{K}$, we obtain a new manifold $\bar{M}_{K}$ such that $\partial \bar{M}_{K}$ consists of a finite number of open annuli, one each for each conjugacy class of parabolic elements in $K$. The manifold $\bar{M}_{K}$ has only one end and it is immediate that $K$ is geometrically finite if the domain of discontinuity of $K$ is nonempty. Thus, whether $K$ has parabolics or not, Theorem 1 follows from the next assertion.

\section{Assertion. The domain of discontinuity of $K$ is nonempty.}

To prove the above assertion, we need a description of the map $j: B^{2} \rightarrow B^{3}$ given in [C-T], and its extension to the case when there are parabolics. Let $\lambda_{s}$ and $\lambda_{u}$ be the stable and unstable laminations on $S$ given by the fibration of $M$ (see $\left[\mathrm{Th}_{1}\right]$ ) and let $\lambda_{s}^{\sim}$ and $\lambda_{u}^{\sim}$ be the lifts of $\lambda_{s}$ and $\lambda_{u}$ to $H^{2}$. Now, consider the 2-sphere $S^{2}$ as the union of two copies $B_{-}^{2}$ and $B_{+}^{2}$ of the closed disc $B^{2}=H^{2} \cup S_{\infty}^{1}$ intersecting along the common boundary $S_{\infty}^{1}$. We take a copy $\lambda_{1}$ of $\lambda_{s}^{\sim}$ on $B_{-}^{2}$, a copy $\lambda_{2}$ of $\lambda_{u}^{\sim}$ on $B_{+}^{2}$. These give a cellular decomposition $S^{2}\left(\lambda_{1}, \lambda_{2}\right)$ of $S^{2}$ as follows: An element $g$ of $S^{2}\left(\lambda_{1}, \lambda_{2}\right)$ is either (i) the closure in $B_{-}^{2}$ of a component of $B_{-}^{2}-\lambda_{1}$, or (ii) the closure in $B_{-}^{2}$ of a leaf of $\lambda_{1}$ not contained in an element of type (i), or (iii) the closure in $B_{+}^{2}$ of a component of $B_{+}^{2}-\lambda_{2}$, or (iv) the closure in $B_{+}^{2}$ of a leaf of $\lambda_{2}$ not contained in an element of type (iii), or (v) a singleton on $S_{\infty}^{1}$ not contained in any of the above.

If there are not parabolics in $G$, components of type (i) and (iii) above are finite sided and no two elements of $S^{2}\left(\lambda_{1}, \lambda_{2}\right)$ intersect. The decomposition of $S^{2}$ by $S^{2}\left(\lambda_{1}, \lambda_{2}\right)$ yields again a 2-sphere that we identify with $S_{\infty}^{2}$, and the restriction of the decomposition map $\pi$ to $S_{\infty}^{1}$ is equivalent to the CannonThurston map $J_{\infty}: S_{\infty}^{1} \rightarrow S_{\infty}^{2}$. If there are parabolics in $G$, the lamination 
$\lambda_{s}$ (resp. $\lambda_{u}$ ) does not pass through the punctures of $S$, and each puncture of $S$ is contained in exactly one component of $S-\lambda_{s}$ (resp. $S-\lambda_{u}$ ) with infinite cyclic fundamental group. Thus $H^{2}-\lambda_{s}^{\sim}$ (resp. $H^{2}-\lambda_{u}^{\sim}$ ) has infinite sided components, one such for each parabolic fixed point of $H^{\prime}$. The sides of the polygon are all translates of finite numbers of them by the infinite cyclic subgroup of $H^{\prime}$ corresponding to the puncture and thus the sides converge to the parabolic fixed point: We now have for each parabolic fixed point $p$ of $H^{\prime}$ and element $g_{1}^{p}$ in $B_{-}^{2}$, an element $g_{2}^{p}$ in $B_{+}^{2}$ both infinite sided and intersecting exactly at $p$ and except for such no two elements $S^{2}\left(\lambda_{1}, \lambda_{2}\right)$ intersect. The decomposition is again cellular and the map $j_{\infty}: S_{\infty}^{1} \rightarrow S_{\infty}^{2}$ is given as before. Whereas $j_{\infty}$ is finite-to-one in the purely hyperbolic case, each parabolic fixed point $p$ is identified under $j_{\infty}$ with an infinite number of other points, namely the vertices of $g_{1}^{p}$ and $g_{2}^{p}$. We now make an assertion.

3. Assertion. The map $j_{\infty}$ is one-to-one on the fixed points of hyperbolic elements of $H^{\prime}$.

To prove the claim, observe that $j_{\infty}(x)=j_{\infty}(y)$ if and only if (a) $x$ and $y$ are the vertices of a polygon of $\lambda_{1}$ or $\lambda_{2}$; or (b) there is a parabolic fixed point $p$ of $H^{\prime}$, and $\{x, y\} \subset\{p\} \cup\left\{\right.$ vertices of $\left.\lambda_{1}^{b}\right\} \cup\left\{\right.$ vertices of $\left.\lambda_{2}^{b}\right\}$. So, it is enough to show that if $x$ is the fixed point of a hyperbolic element $g$ of $H^{\prime}$, then $x$ is not the endpoint of a leaf of $\lambda_{s}^{\sim}$ or $\lambda_{u}^{\sim}$. Suppose that $x$ is the endpoint of a leaf $\lambda$ of $\lambda_{s}^{\sim}$ and consider the axis $A_{g}$ of $g$. Since there are no closed geodesics in $S-\lambda_{u}$, we see that $A_{g}$ has to intersect a leaf $\mu$ of $\lambda_{s}^{\sim}$. Then, either $g^{n}(\mu)$ or $g^{-n}(\mu)$ converges to $x$ as $n \rightarrow \infty$. This, in turn, implies that for sufficiently large $n, g^{n}(\mu)$ or $g^{-n}(\mu)$ intersects $\lambda$, which is a contradiction since both are parts of $\lambda_{s}^{\sim}$. The same argument works for $\lambda_{u}^{\sim}$ as well and proves Assertion 3.

We will next prove claim 2 using the above assertion. Recall that $K^{\prime}=$ $h_{*}^{-1}(K)$ is of infinite index in $H^{\prime}$, and that the limit set of $H^{\prime}$ is all of $S_{\infty}^{1}$. By a result of Greenberg [G] the limit set $\Lambda\left(K^{\prime}\right)$ of $K^{\prime}$ cannot be the whole of $S_{\infty}^{1}$. Since the fixed points of axes of hyperbolic elements of $H^{\prime}$ are dense in $\Lambda\left(H^{\prime}\right)=S_{\infty}^{1}$, we can find $x \in S_{\infty}^{1}-\Lambda\left(K^{\prime}\right)$ that is the fixed point of a hyperbolic element $g \in H^{\prime}-K^{\prime}$. We claim that $j_{\infty}(x)$ does not belong to $\Lambda(K)$. Otherwise, let $j_{\infty}(x) \in \Lambda(K)$. Since $j_{\infty}$ is equivariant, $j_{\infty}\left(\Lambda\left(K^{\prime}\right)\right)=$ $\Lambda(K)$ and thus $j_{\infty}(x)=j_{\infty}(y)$ for some $y \in \Lambda\left(K^{\prime}\right)$. On the other hand, by Assertion 3, $x$ is not identified with any other point under $j_{\infty}$. Hence, the limit set of $K$ does not fill up $S_{\infty}^{2}$ and as noted in the beginning, this is enough to conclude the geometric finiteness of $K$.

\section{REFERENCES}

[Bo] F. Bonahon, Bouts des varietes hyperboliques de dimension 3, Ann. Math. 124 (1986), 71-158.

[C-T] J. Cannon and W. Thurston, Group invariant Peano curves, preprint, 1986. 
[F] S. Fenley, Ph.D. thesis, Princeton, 1989.

[G] Leon Greenberg, Discrete groups of motions, Canad. J. Math. 12 (1960), 415-426.

[M] B. Maskit, On free Kleinian groups, Duke Math. J. 48 (1981), 755-765.

[Sc1] P. Scott, Subgroups of surface groups are almost geometric, J. London Math. Soc. 17 (1978), 555-565.

[Sc2] _ Subgroups of surface groups are almost geometric-correction, J. London Math. Soc. 32 (1985), 217-220.

$\left[\mathrm{Th}_{1}\right] \mathrm{W}$. Thurston, Hyperbolic structures on 3-manifolds II, Surface groups and 3-manifolds which fibre over the circle, preprint, 1987.

$\left[\mathrm{Th}_{2}\right]$ - Notes on hyperbolic geometry, Princeton, NJ, preprint.

Department of Mathematics, The University of Michigan, Ann Arbor, Michigan 48109

Department of Mathematics, The University of Melbourne, Parkville, 3052, AusTRALIA 